\title{
THE FORMATION OF THE GALACTIC BULGE
}

\author{
M. SAMLAND AND G. HENSLER \\ Institut für Astronomie und Astrophysik \\ Universität Kiel, D-24098 Kiel, Germany
}

The question is adressed whether the problem of the stellar metallicity distribution and the dynamics of the stellar components in the bulge as found out by refined observations during recent years can be understood within the context of the evolution of the whole Galaxy. A selfconsistent galaxy model has to explain the apparent differences in the effective yields of the enrichment of bulge, disk and halo. Moreover, it has to account for the observed age-metallicity distribution of the stellar components. It appears that chemo-dynamical infall models provide a consistent description of the bulge, in particular the metallicity distribution of the $\mathrm{K}$ giants. It should be emphasized that simple closed-box models are not appropriate, because during the bulge formation there is infall of cloudy medium (CM), as well as outflow of hot, ionized gas ejected by supernovae type II (SNII). Therefore dynamical processes have to be taken into account. For details of the chemodynamical description we refer to Samland \& Hensler (1994) and references therein.

The chemo-dynamical models show that the early metal enrichment of the interstellar medium in the bulge by means of SNII remains initially confined to the hot gas phase because of the inefficiency of phase mixing. Since the SNII also heat the intercloud medium (ICM), a SN-driven wind sets in, so that the enriched gas leaves to some extent the bulge region and enriches the outer parts of the galaxy. Since the clouds embedded in the ICM evaporate, the outflowing gas has a metallicity which is nearly solar. Fig. 1 shows the infall rate of the CM, the outflow rate of the ICM and the sum of both processes for the central $2 \mathrm{kpc}$. It is obvious, that most of the bulge mass is accreted in the period of $2 \cdot 10^{9}-8 \cdot 10^{9}$ years. During the same time the iron abundance in the $\mathrm{CM}$ increases from $[\mathrm{Fe} / \mathrm{H}]=-1.0$ to $[\mathrm{Fe} / \mathrm{H}]=0.5$ and due to an efficient star formation most of the $\mathrm{CM}$ is converted into stars.

Fig. 2 shows the calculated iron distribution of $\mathrm{K}$ giants in comparison with the data of Rich (1988) (left) and the corrected data of McWilliam \& 


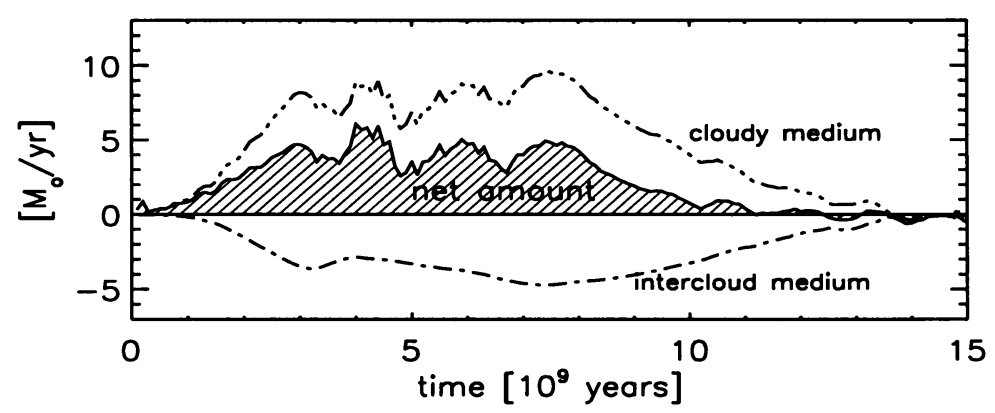

Figure 1. Infall rate of cloudy medium and outflow rate of intercloud medium for the central $2 \mathrm{kpc}$ of the galaxy. The hashed area indicates the net effect of both processes.
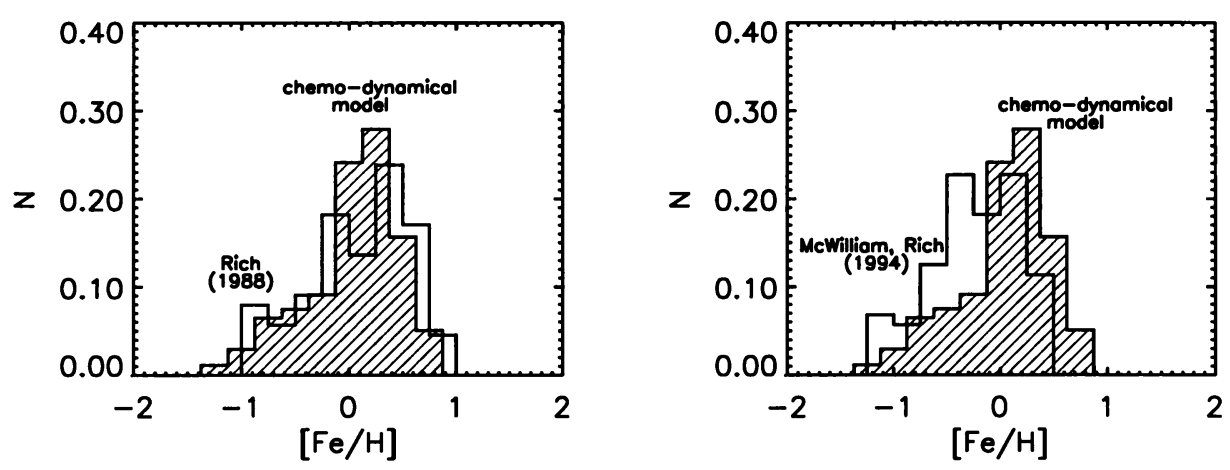

Figure 2. Abundance distribution of $K$ giants in Baade's window in comparison with the distribution derived from a chemo-dynamical model (hatched area). The solid histogram outlined in the left diagram represents the original data from Rich (1988) and in the right diagram the corrected data by McWilliam \& Rich, (1994).

Rich (1994) (right). Taking into account the uncertainities in the observational data and that the correction formula of McWilliam \& Rich is only a rough fit, the model agrees convincingly well with the observations.

Moreover, the chemo-dynamical model explains the $\mathrm{Fe}, \mathrm{N}$ and $\mathrm{O}$ distributions of bulge, halo and disk stars (Samland \& Hensler, 1994). In contrast to the box-models, the chemo-dynamical models are self-consistent and there is no need of ad-hoc assumptions (e.g. different yields for bulge, disk and halo stars) to explain the stellar metallicity distributions.

\section{References}

McWilliam, A., Rich, R.M., (1994), Astrophys. J. Suppl. 91,749

Rich, R.M., (1988), Astron. J. 95,828

Samland, M., Hensler, G., (1994), Metal Pre-Enrichment of the Galactic Disk in: IAU

Symp. No. 169, Unsolved Problems of the Milky Way, ed. L.Blitz 